Cardiology 1971-72;56:1

\title{
Acute Hypoxia
}

Chairmen: W. F. H. M. Mommaerts and G. Majno Rapporteurs: H. A. Heggtveit and J. Scheuer 